\title{
La puntura a occhiello o buttonhole: quali sono le cose che "fanno la differenza". Una revisione della letteratura infermieristica
}

\author{
Marisa Pegoraro \\ Azienda Ospedaliera Ca' Granda Niguarda, Milano, EDTNA/ERCA, Filiale Italiana
}

\begin{abstract}
ButTONHOLE: What MAKes THE DIFFERENCE
Abstract. In the last 10 years a great number of contributions have been published on the buttonhole (BH) cannulation technique, mainly by nurses. The word "buttonholing" has gained popularity in the dialysis settings. All studies tried to explore the announced attracting outcomes of this technique: minor pain, less aneurisms, less AVF complications, and easier cannulation for both nurses and patients. Data have been collected for all these areas either as single topic or in multiple-topic data collection analysis, in different settings, with different sample sizes and different observational times. Thanks to this "realistic" approach the challenge of infection could be detected and managed. The variation in the incidence of infections had very low statistical impact, but was relevant for the caring team. What was cited as a "rare alert" by BH founders showed to be, in fact, a real possibility. The present article will refer to the most recent studies, all the variables included, and the related outcomes, and will then try to highlight the issues, related to nursing care, which correlate with positive outcomes of $\mathrm{BH}$ performance in hemodialysis patients.
\end{abstract}

Key words: Dialysis patient, Arteriovenous fistula, Buttonhole cannulation, Vascular access nurse

Conflict of interest: None.

Ricevuto: 5 Maggio 2013; Accettato: 28 Maggio 2013

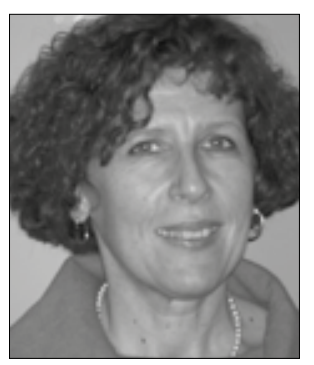

Marisa Pegoraro

\section{Introduzione}

I dati di evidenza scientifica raccolti in questo ultimo decennio dallo studio DOPPS (1) hanno confermato la fistola nativa (FAV) come accesso vascolare (AV) di elezione per i pazienti in trattamento emodialitico. Questo dato ha messo in moto un cambiamento delle pratiche cliniche soprattutto in quei Paesi dove la FAV era poco utilizzata (Nord America, UK, Australia) (2). I professionisti di questi Paesi hanno dovuto "ri-scoprire" come pungere le FAV (3), i vari metodi di puntura e le caratteristiche di ognuno (4). La puntura a sito costante ha raccolto molto interesse e favori presso gli infermieri a seguito delle osservazioni empiriche riportate da coloro che la praticavano da molto tempo (5). Con l'aumentare dell'utilizzo, oltre che le positività sono anche sorte le osservazioni sulle aree di criticità.

I dati provenienti da alcuni studi randomizzati controllati (RCT) $(6,7)$ sono stati in controtendenza rispetto a quanto atteso e rispetto agli studi osservazionali disponibili. In alcuni, la tecnica a sito costante apporta una complessiva positività e le infezioni sono un evento marginale e statisticamente non rilevante (8); in altri, i dati sono piuttosto negativi su vari fronti $(6,7)$, pur non raggiungendo una significatività statistica. Sorge spontanea la domanda: perché una tecnica, eseguita con protocolli apparentemente simili, conduce a esiti diversi? Quali sono le variabili che fanno la differenza? Le variabili sensibili sono chiare e dichiarate o, piuttosto, "occulte"?. È possibile che elementi considerati piuttosto marginali rispetto al "protocollo" tecnico risultano importanti rispetto agli esiti? L'obiettivo della presente revisione della letteratura si propone di evidenziare quali siano le componenti di natura assistenziale e clinico-organizzativa correlabili a esiti positivi nell'applicazione della tecnica di puntura a sito costante (puntura a occhiello o buttonhole o $\mathrm{BH}$ ).

\section{I principi dell'“occhiello"}

Ripassiamo insieme i cardini del metodo di puntura delle FAV NATIVE a SITO COSTANTE od occhiello.

- Definizione dei siti: cute integra, facile repere, perpendicolare al vaso. Se la FAV è di vecchia data, evitare gli aneurismi e, se impossibile, scegliere la base della dilatazione dove maggiore è l'integrità strutturale della cute e del vaso 
- Lavaggio del braccio FAV appena prima della seduta, per abbattere la flora batterica residente.

- Disinfezione/rimozione della crosta con appositi ausili, evitare strumenti taglienti, se possibile.

- Ri-disinfezione del sito dopo la rimozione della crosta.

- Creazione del percorso sottocutaneo: se si utilizza il metodo manuale, necessita di un solo incannulatore per almeno 3-4 settimane (se impossibile organizzativamente, massimo 2 persone). Più si è precisi in questa fase, più sarà preciso il percorso sottocutaneo e minori saranno i problemi nel mediolungo periodo. In alternativa, si possono utilizzare gli ausili come il sistema "bio-hole ${ }^{\mathrm{TM}}$ ' (9) puntina in policarbonato o l'agocannula (10).

- Metodo di incannulazione del vaso: è preferibile il metodo "touch cannulation", presa delicata dal tubicino, soprattutto quando si usa l'ago smusso (11).

\section{La letteratura sul buttonhole}

L'approfondimento bibliografico è stato fatto consultando le banche dati di CINHAL e MEDLINE, via Metacrawler, con il seguente P.I.C.O.: "dialysis" OR "dialysis patient" AND "arteriovenous fistula" AND "cannulation" AND "buttonhole cannulation". Alla ricerca elettronica è stato associato un controllo incrociato sulle referenze citate nei vari articoli. Sulla puntura a sito costante della FAV nativa sono stati complessivamente trovati 56 lavori, pubblicati dal 1979 al 2012. La letteratura reperita (al Febbraio 2013) non contemplava le presentazioni a congressi locali, nazionali e internazionali, che farebbero salire le citazioni ampiamente oltre il centinaio. Dei lavori trovati, 3 sono Linee Guida e 21 sono studi

TABELLA I - PROVENIENZA DEGLI STUDI PRIMARI

\begin{tabular}{|ll|ll|ll|}
\hline Asia & 2 & Australia & 2 & Brasile & 3 \\
\hline Canada & 6 & Eu & 5 & Usa & 3 \\
\hline
\end{tabular}

TABELLA II - PROVENIENZA E TIPOLOGIA DEGLI STUDI SECONDARI

\begin{tabular}{lccc}
\hline Tipologia & Numero & Provenienza & Affiliazione \\
\hline Review & 1 & Usa & Anna \\
& 1 & Canada & Cannt \\
& 2 & Uk & Edtna/Erca \\
Continuous Education & 2 & Aus & Rsa \\
Report & 5 & Usa & Anna \\
& 5 & Usa & Anna \\
\hline
\end{tabular}

ANNA: American Nephrology Nurses Association

CANNT: Canadian Association of Nephrology Nurses and Technicians EDTNA/ERCA: European Dialysis and Transplant Nurses Association - European Renal Care Association

RSA: Renal Society of Australasia primari consistenti in raccolte dati eseguite nelle varie realtà operative, di cui 3 RCT, 8 studi di coorte, 8 prospettici, 3 retrospettivi e 2 inchieste qualitative. I 17 studi secondari sono composti da revisioni di articoli primari, articoli di formazione continua e raccolte di approfondimento; 15 sono lettere a rubriche, pareri di esperti e valutazioni sugli esiti citati negli studi primari, che sono stati esclusi dall'approfondimento. Le aree di provenienza dei lavori primari sono piuttosto bilanciate tra i vari continenti (Tab. I). I lavori secondari sono, invece, in maggioranza prodotti nei Paesi di cultura anglosassone (Tab. II), da Autori affiliati alle società scientifiche locali o internazionali.

Volendo considerare le argomentazioni e i contenuti dei lavori analizzati, si colgono simmetrie di ricerca che convergono su alcune aree affrontate di volta in volta, in modo dipendente, indipendente $o$ in relazione ai criteri di convenienza specifici del contesto in cui venivano svolte le ricerche. Le argomentazioni comuni di ricerca e raccolta dati sono raggruppabili (Tab. III) come segue:

- Il dolore alla puntura, rilevato dai pazienti

- L'insorgenza di complicazioni non infettive:
○ Aneurismi
- Ematomi
- Emostasi/sanguinamenti

- Difficoltà nell'incannulazione rilevata dagli infermieri

- L'insorgenza di complicazioni infettive locali o generali

\section{Analisi dei dati}

\section{- Il dolore alla puntura}

Questa componente è stata generalmente valutata con una Scala Visiva Analogica (VAS) o iconografica (WongBaker). Figueiredo (12) descrive molto accuratamente gli elementi che rendono il "dolore" poco comparabile e sottoposto a una serie di elementi tecnici e relazionali che concorrono agli esiti, non ultimo il tipo di relazione con l'infermiere o il personale. Il genere femminile e il $2^{\circ}$ ago inserito sono correlati con una maggiore rilevazione dolorosa. La maggioranza degli studi rileva una diminuzione della percezione del dolore da parte dei pazienti (9, 12-18) punti con tecnica ad occhiello e, quando richiesto,

TABELLA III - SINTESI DELLE CITAZIONI PER AREA DI RICERCA, NEI 21 ARTICOLI PRIMARI ANALIZZATI

\begin{tabular}{lccc}
\hline Area indagata & Totale citazioni & $\begin{array}{c}\text { Diminuito } \\
\text { in } \mathbf{~ B H}\end{array}$ & $\begin{array}{c}\text { Aumentato } \\
\text { in } \mathbf{~ B H ~}\end{array}$ \\
\hline DOLORE & 12 & 10 & 2 \\
EMATOMI & 8 & 7 & 1 \\
ANEURISMI & 8 & 7 & 1 \\
EMOSTASI & 10 & 9 & 1 \\
DIFFICOLTÀ & 13 & 8 & 5 \\
INFEZIONI & 17 & 4 & 13 \\
\hline
\end{tabular}


una predilezione degli stessi verso questa tecnica $(12,15$, 16). In altri casi $(6,8)$, le differenze di percezione del dolore non hanno raggiunto la significatività statistica, seppure $\mathrm{i}$ pazienti, o gli infermieri, abbiano poi riferito un minore uso di anestetico locale. L'utilizzo di anestetico locale sembra molto comune nei centri dialisi d'oltreoceano $(6,7)$ e quasi inesistente (19) o molto raro in altre realtà. In alcuni casi, il persistente utilizzo dell'anestetico ha reso non comparabili i dati raccolti (7). La diminuzione del dolore ha permesso ai pazienti rapportarsi più positivamente con la possibilità di passare alla dialisi domiciliare $(14,20)$. Verhallen $(21)$ ritiene che utilizzare prima l'incannulazione a rotazione dei siti sia utile per dare ai pazienti maggiore consapevolezza dei benefici del sito costante, ma, nella discussione riferita da Marcutt (5), Boogaerts ritiene che sia antietico procurare dolore quando si potrebbe evitare. VanLoon (22) ritiene che i pazienti punti da subito in un sito costante abbiano indicato scale di dolore maggiori proprio per l'impossibilità di fare confronti con altre tecniche. E stata rilevata una diminuzione del dolore dopo 3-6 mesi, cioè quando il sito si è completamente assestato e i livelli di ansia sono diminuiti $(13,16,18$, 21). Sono stati sporadicamente riportati casi di persistenza del dolore anche durante il trattamento emodialitico (6).

Evidenza: la tecnica a sito costante concorre a diminuire il dolore, anche se la valutazione di questo beneficio è poco predittibile né oggettivamente valutabile, data la soggettività della percezione e la diversità dei contesti clinici.

\section{- Ematomi e infiltrazioni}

L'inserimento scorretto dell'ago nella vena può dare origine a uno stravaso di sangue intorno alla stessa, tale da rendere le successive incannulazioni difficili, concorrendo a un precoce malfunzionamento e a una limitata sopravvivenza della FAV $(22,23)$. Questa possibilità è molto elevata con l'uso dell'ago tagliente, nei pazienti anziani e nei primi mesi di utilizzo delle fistole (24). Nei dati raccolti si rileva una diminuzione degli ematomi nella puntura a occhiello, dopo essere passati all'utilizzo degli aghi smussi $(6-8,15)$. L'utilizzo dell'ago smusso è correlato con una maggiore difficoltà di incannulazione, ma con una minore insorgenza di ematomi (22). Le incannulazioni non riuscite, senza la formazione dell'ematoma, sono state incluse in alcuni studi tra gli eventi avversi $(6,25)$ della puntura a occhiello e in altri citati come "effetto trampolino" (26). L'incannulazione efficace è correlata con l'ottimale conformazione del sito e con le abilità/esperienza degli infermieri $(6,13)$ o del paziente nel caso di auto-puntura $(21)$. È un evento che tende allo " 0 " con l'aumentare della pratica $(15$, $16,19)$ e con il persistente uso dell'ago smusso. Solo nello studio di Chow (6) si sono rilevati più ematomi e infiltrazioni, rispetto al gruppo in rotazione dei siti, ma lo stesso Autore ha messo questo dato in correlazione con la poca esperienza del personale, che non aveva mai praticato prima la puntura a sito costante, e con il numero di incannulazioni eseguite.

Evidenza: la puntura a sito costante ha indotto mediamente meno ematomi e sanguinamenti a seguito della puntura della FAV. L'uso dell'ago smusso ha limitato ulteriormente questi eventi.

\section{- Aneurismi}

Questo è un parametro che non è stato sempre valutato, in parte perché diventa evidente nel medio-lungo periodo e gli studi disponibili hanno avuto una durata relativamente breve, in parte perché riguarda la sfera di percezione corporea personale correlata a cultura e ambiente. Negli studi brasiliani $(16,17)$, l'elemento estetico era un parametro evidente, dati il clima e le braccia scoperte. In una fase iniziale, la dilatazione è ritenuta funzionale per una facile infissione degli aghi $(21,22)$. L'aneurisma non deve essere mai punto sulla sua cuspide, ma solo alla base, dove maggiore è la resistenza strutturale (14). Nella puntura a sito costante, poichè l'ingiuria é concentrata in una piccola area, la struttura tissutale del vaso e del sottocute resta integra. Ne consegue che la formazione di aneurismi è significativamente diminuita con la puntura a occhiello $(8,15,21,22)$. Negli studi che hanno riportato l'utilizzo del sito costante sulle FAV aneurismatiche è stata rilevata una diminuzione, statisticamente significativa, del diametro strutturale e visivo dell'aneurisma $(14,15,19)$. La formazione di aneurismi, pur in presenza di una puntura a sito costante, è stata messa in relazione con l'uso degli aghi taglienti (5) e con il possibile duplice effetto di sfilettamento della parete interna del vaso, a seguito dei movimenti del paziente, e di mini puntura ad area data dall'impreciso inserimento dell'ago tagliente nel sito cicatriziale. Hsiao non ha rilevato formazioni stenotiche (27), anche se era presente una modesta dilatazione (1-2 $\mathrm{mm})$ in prossimità del sito.

Evidenza: la puntura a sito costante, eseguita con un ago smusso, non favorisce la formazione di aneurismi o concorre a un loro contenimento.

\section{- Sanguinamenti}

Durante il trattamento dialitico, si utilizzano varie tipologie di eparina per mantenere pervio il circuito extracorporeo. Negli studi analizzati non è mai stato apportato alcun cambiamento al regime anticoagulante del paziente. La maggioranza degli Autori ha rilevato una diminuzione dei tempi di emostasi a fine trattamento $(9,14,15,17,19,21,28)$ e, negli altri casi, un tempo di emostasi simile alla metodica tradizionale ( 7 , 18). L'emostasi si ottiene comprimendo con un tampone il punto di inserzione, dopo la rimozione dell'ago. Il tempo di compressione si colloca intorno a $\pm 5^{\prime}$ ( $\mathrm{min}$ ) per $1{ }^{\prime} 80 \%$ circa dei pazienti e solo per una piccola frazione a un tempo $\geq 10$ '. Anche Kim (18) ha confermato una diminuzione dei tempi di emostasi, pur riportando range di controllo significativamente diversi $\left(<15^{\prime},<30^{\prime},>30^{\prime}\right)$, rilevando che, con il sito costante, il $60 \%$ dei pazienti raggiungeva la completa emostasi prima dei $15^{\prime}$ e nessuno oltre i 30 '. Una variabile citata rispetto all'emostasi è stata la perdita di sangue intorno all'ago durante il trattamento, evento che accade molto raramente con l'uso degli aghi smussi (13, 22) che, non lacerando la cute, provocano meno distruzione tissutale. Sono stati riportati eventi locali in concomitanza con l'uso dell'ago tagliente (6).

Evidenza: nonostante le ripetute punture sullo stesso sito, la puntura a occhiello eseguita con aghi smussi non aumenta $i$ tempi di emostasi, ma tende, piuttosto, a diminuirli. 


\section{- Difficoltà nell'incannulazione con ago smusso}

Questo argomento è correlato al modo in cui il sito viene creato, alla manualità con l'ago smusso e alle caratteristiche del paziente. Il protocollo originale (29) prevedeva un solo incannulatore fino alla completa definizione del sito e il definitivo uso dell'ago smusso. Alcuni Autori hanno riportato una variazione a questa indicazione $(7,14,22)$, ampliando il numero di operatori in questa fase, a seguito delle difficoltà a far coincidere la turnistica infermiere/paziente. Altri hanno definito come cruciale la presenza di 1 solo incannulatore sempre per lo stesso paziente, durante il periodo di preparazione del sito $(16,19,30)$. Quando l'operatore specifico non era in servizio, la FAV veniva punta a rotazione dei siti, da un altro infermiere. È stato notato come le difficoltà nell'introduzione dell'ago smusso avvengono più spesso dopo il turno interdialitico più lungo (19), mentre sono praticamente assenti nei trattamenti di ED giornaliera, dove il sito è punto tutti i giorni. Marticorena (14) nella versione modificata, cita una precisa suddivisione della competenze tra il gruppo dei "primi incannatori, quelli di "secondo livello" e il resto dello staff. L'uso dell'ago tagliente in modo estemporaneo è sconsigliato, una volta che si è assestato l'uso dell'ago smusso (22). Nello studio di Labriola, l'uso dell'ago tagliente era abilitato solo per gli Infermieri Referenti e totalmente vietato al resto del personale (30). La poca esperienza all'uso dell'ago smusso è correlata con la frequenza di problemi all'incannulazione $(6,7,16,17)$. Esiste una "curva di apprendimento" all'utilizzo dell'ago smusso: il personale con meno esperienza e i periodi iniziali di applicazione sono più problematici. Al problema è stato ovviato procurando delle foto digitali del corretto angolo di ingresso nella cute (30), abbinate al tutoraggio per le prime incannulazioni di "altri operatori” (14) e per l'inserimento dell'ago smusso con "touch cannulation" (11). La specificità di applicazione dell'ago smusso a volte è stata considerata una positiva acquisizione di competenze $(17,19)$.

Evidenza: le difficoltà della puntura con ago smusso diminuiscono con la pratica clinica e con l'aumentare delle incannulazioni.

\section{- Complicanze infettive}

Il Registro americano Renal Data System (2006) riportava un'incidenza di 0.6/infezioni/anno/paziente per le FAV e pari a 2.0/infezioni/anno/paziente per i CVC (31). I pazienti nefropatici hanno un sistema immunitario compromesso e l'evento infettivo associato all'accesso vascolare è tra le prime cause di ospedalizzazione e decesso $(31,32)$. Un aumento delle infezioni dell'accesso é stato rilevato in molti studi $(7,9,13,14,116$, $22,30,31,33,34)$, ma solo in un numero limitato di questi (7, $30,31)$ ha raggiunto la significatività statistica. I batteri responsabili sono stati generalmente lo Stafilococco Aureo e S. Epidermidis, comunemente presenti nella flora batterica cutanea, per cui le infezioni sono imputabili a una migrazione batterica e richiedono un'immediata antibioticoterapia (35). Qualche Autore ha ipotizzato che il rischio infettivo potesse annullare i benefici della tecnica $(7,26)$. Contrariamente, altri Autori non hanno rilevato un aumento delle infezioni $(15,18,19,36)$. Nella popolazione di pazienti domiciliari in trattamenti trisettimanali notturni lunghi (6-8 h) e/o notturni quotidiani brevi (3-5 h), l'incidenza di infezioni è risultata maggiore di quanto accadeva in una simile popolazione in dialisi ospedaliera trisettimanale, con puntura a rotazione di siti $(33,37)$. Gli Autori hanno ipotizzato che la frequente e prolungata permanenza degli aghi permettesse la migrazione verso l'interno della flora cutanea. Altri elementi considerati sfavorevoli sono stati il ri-utilizzo di monitor da enuresi (per il controllo delle perdite ematiche accidentali durante il sonno) (33) e la non aderenza al protocollo di disinfezione del sito (37). Anche la semplicità e la facilità della manovra di inserimento degli aghi possono indurre una diminuzione della scrupolosità nella manovra (32) o l'attivazione di comportamenti a rischio da parte dei pazienti, come la rimozione delle croste con strumenti impropri (stuzzicadenti, unghie) (31). Le frenetiche attività di un reparto ospedaliero possono indurre a preparazioni affrettate e superficiali (30), con una incompleta rimozione delle croste (22) o con l'omissione della disinfezione dopo la loro rimozione (6). Labriola (30), forte della ventennale esperienza di sito costante nei pazienti domiciliari e in self-care, nello studio retrospettivo eseguito, cita l'aumento progressivo delle infezioni degli accessi vascolari dopo l'implementazione della tecnica nel reparto ospedaliero. Lo studio retrospettivo ha rilevato come, nonostante la presenza di siti costanti, l'uso degli aghi taglienti interessasse ancora il $48 \%$ dei casi, evidentemente utilizzati quando l'incannulazione con l'ago smusso risultava difficoltosa. La revisione delle procedure e l'allocazione di competenze infermieristiche specifiche a pochi Infermieri Referenti, ridussero l'uso degli aghi taglienti al 2\%. Questo elemento, in concomitanza con un re-training del personale, ha permesso una diminuzione significativa delle infezioni (RR da 2.28 a $0.24 / 1000 \mathrm{gg}$ FAV). La caratteristica che ha allarmato molti servizi dialisi, è stata la comparsa di poche ma gravi batteriemie con infezioni metastatiche $(14,30,37)$, che comparirono a distanza di tempo sia dal periodo di osservazione che dall'evento acuto locale, non imputabili ad altra causa, genesi confermata poi dalla tipologia di germi isolati. Nersharall (37), facendo riferimento alle esperienze della dialisi peritoneale, è riuscito a ridurre gli eventi infettivi da 0.23/1000 gg FAV a 0.005/1000 gg FAV, applicando preventivamene una pomata antibiotica (Muporicin o similari) sul sito dopo l'emostasi e lasciandola asciugare, senza cerotto. Lo stesso Autore riporta l'osservazione di valori elevati di PCR $(>8)$ in tutti i pazienti che hanno sviluppato un'infezione, suggerendo che questa possa essere considerata un fattore di allerta. Marticorena (10), applicando la tecnica a sito costante, con protocollo modificato, su fistole aneurismatiche, ha incluso l'uso cerotti intrisi di soluzioni antibatteriche (betadine o polisporin), da applicare dopo l'emostasi e da tenere in sito per almeno 6 ore. La protezione post dialisi del sito di inserzione, per un certo numero minimo di ore, veniva consigliata anche dagli ideatori della tecnica, Twardosky, Kubara, nel loro primo protocollo (29).

Evidenza: l'applicazione della tecnica deve prevedere un'attenta e costante valutazione delle condizioni operative del personale e dell'igiene del paziente e la supervisione degli esiti. 


\section{Discussione}

\section{- Strategie organizzative}

Dalla letteratura si evince che l'esperienza ha reso la tecnica progressivamente più facile e applicabile: da qui il concetto della "curva di apprendimento" a cui fa riferimento VanLoon (22). Negli ambienti ospedalieri con un grande numero di infermieri sono state applicate delle strategie a supporto. Alcune di queste sono già state citate, come, per esempio, la scelta del gruppo di “primi incannulatori” per Marticorena (14), l'uso del tagliente solo per gli Infermieri Referenti per Labriola (30) e la strutturazione dei turni infermieristici a giorni alterni come cita Ball (13). La semplice applicazione di un'antibioticoterapia locale ha prevenuto situazioni gravi nell'esperienza di Nersharall (37) e di Marticorena $(10,14)$. Nella revisione di Marcutt (5), il contributo di Boogaerts indica l'allocazione delle FAV in 3 fasce di difficoltà (facile, complesso, difficile), che ne determinava l'accessibilità alla puntura: maggiore è la difficoltà, minore e più qualificato e limitato è il numero degli infermieri che vi potevano accedere. Mott \& Prowant (11) hanno suggerito la presa dell'ago per il tubicino (touch cannulation), che, con delicatezza, permette all'ago smusso di percorrere autonomamente il tragitto con meno resistenze. Ball (38) allerta rispetto all'introflessione del sito (hubbing), indicando di non inserire l'ago completamente, lasciando fuori alcuni $\mathrm{mm}$ di cannula, in modo che la parte conica di raccordo, solitamente più larga della cannula nell'ago, non spinga/trazioni l'exit. Inoltre, l'introflessione rende difficile la completa rimozione delle croste con la conseguente permanenza di residui tissutali potenzialmente pericolosi. Bircherought (32) suggerisce l'utilizzo di una scheda di osservazione da compilare ad ogni sessione e dei report mensili di audit sugli accessi vascolari fatti con tutto il personale della dialisi. L'estensione delle iniziative al personale è specificatamente citato in riferimento alla figura del "tecnico sanitario" (health care technician) che, nei centri del Nord America, partecipa direttamente alla gestione della seduta e del paziente ed è coinvolto anche nelle fasi di incannulazione (26). L'informazione e l'aggiornamento periodico del personale è stato citato come essenziale, soprattutto quando il contesto è grande e laborioso $(14,19,22,30)$ o quando si devono cambiare/rinnovare le pratiche cliniche $(6,39)$.

Nella letteratura analizzata non emergono delle preclusioni all'uso della tecnica di puntura a occhiello rispetto a certe tipologie di pazienti particolarmente fragili (anziani, diabetici, immunodepressi). Si segnala, piuttosto, una crescente consapevolezza rispetto alla fase di "detersione, rimozione della crosta e ri-disinfezione" del sito con la messa a punto di strategie volte a limitare le micro ingiurie tissutali del sito esterno. Alcuni Autori propongono una procedura di detersione tipo "scrub" con sapone detergente, specificatamente sul sito, con spugnette di normale uso cosmetico (40). Più che limitare la tecnica a certi pazienti, che magari ne godrebbero particolarmente, si ricercano pratiche cliniche efficaci e idonee alla specifica tipologia di pazienti. Questo indica che più i pazienti sono "fragili", più le manovre assistenziali dovrebbero essere "delicate \& fisio-compatibili". La ricerca clinica è in continua analisi e revisione.

\section{- Vascular Access Nurse}

Definire il peso di tale figura di competenza avanzata non è tra gli obiettivi del presente elaborato; malgrado ciò, poiché la figura è stata direttamente o indirettamente citata nei vari articoli, in concomitanza con progetti di "quality improvement" o di ricerca clinica, la sua citazione è doverosa nell'ambito del contesto affrontato in questo elaborato.

La necessità di migliorare l'efficienza nella gestione assistenziale degli accessi vascolari, in senso lato, e, specificatamente, di migliorare le competenze di incannulazione e di monitorare l'andamento delle infezioni in FAV e CVC ha indotto, nei servizi dialisi dei Paesi del Nord America e del Nord Europa, la definizione di Vascular Access Nurse (41) o Infermiere Referente degli Accessi Vascolari.

In letteratura, la creazione del "Vascular Access Nurse" viene descritta come un percorso di competenza clinica, di efficienza organizzativa, di supervisione, di monitoraggio e di gestione complessiva degli accessi vascolari (42). Una figura che costituisce una variabile rilevante ai fini degli esiti $(43,44)$. Nei lavori di origine anglosassone si menzionano, direttamente o indirettamente, delle figure infermieristiche con competenze avanzate, acquisite attraverso corsi interni o percorsi certificati di apprendimento. Queste figure hanno ruoli codificati e riconosciuti, all'interno del servizio, con responsabilità rispetto alla formazione, alla ricerca, al controllo e alla supervisione dei protocolli e alla dialettica interprofessionale rispetto agli obiettivi e agli esiti del trattamento, strumenti essenziali per l'approccio clinico basato sulle evidenze. Nessuna delle ricerche pubblicate sarebbe stata possibile in assenza di tali figure, nell'ambito del gruppo infermieristico.

In Italia, le figure di competenza infermieristica avanzata, certificata e codificata nella pratica clinica, non sono ancora una realtà. Alcuni rari casi citati nella bibliografia locale (45) rilevano comunque un crescente interesse e la necessità della definizione di tali figure di competenza clinica avanzata, tra cui quella dell'Infermiere Referente degli Accessi Vascolari. Ai fini della gestione della tecnica di puntura a occhiello, la presenza di questo ruolo di competenza è fondamentale ai fini degli esiti. È un fattore strategico non ancora conosciuto o acquisito, a livello nazionale, anche se imprescindibile per poter fare una comparazione dei dati di esito.

Non c'è dubbio, infatti, che i percorsi avanzati creino quella particolare condizione definita come "accountability", in cui la "responsabilità" è correlata alla "capacità" di potersi assumere l'onere o l'onore delle conseguenze. In un ambito in cui tutti fanno potenzialmente tutto, chi è responsabile di specifiche azioni? Chi si assume l'onere di attivare una nuova pratica clinica in assenza di una definizione di ruolo (job description)?

In un buon numero dei contributi pubblicati (40\%), gli Autori citano di avere utilizzato fondi allocati alla ricerca clinica proveniente o dalle istituzioni universitarie presso cui lavoravano o da fondi privati o da borse di studio. Indipendentemente dalla provenienza, il fatto che la ricerca sia commisurata a un valore economico è anch'esso cosa poco nota nella nostra realtà nazionale. 


\section{Conclusioni}

Rispetto all'obiettivo di evidenziare quali siano le "cose che fanno la differenza" di esito nell'utilizzo del buttonhole, nei pazienti in emodialisi, è emersa una serie di componenti che non sono sempre semplici azioni, ma piuttosto, a volte, complesse strategie di osservazione e riflessione, che attivano il dibattito locale (breefing) (32), la pubblicazione e il confronto dei risultati (benchmarking) (13). I dati emersi suggeriscono delle strategie organizzative che esaltano pratiche clinico-organizzative attente e adattabili ai diversi contesti quali:

- Modifiche della turnistica $(13,30)$ degli Infermieri Referenti o primi incannulatori o referenti degli accessi vascolari, in relazione alla complessità assistenziale da affrontare;

- Attivazione di pratiche cliniche alternative:

- Punture su altri siti, quando fosse assente la persona dedicata $(19,22)$

- Lavaggio del braccio appena prima della seduta (14)

- La "touch cannulation" (11)

- Le pratiche volte a evitare 1'“hubbing" (38);

- Dualità e progressività nella divulgazione delle competenze di incannulazione tra i vari professionisti $(5,14)$;

- Utilizzo di esperienze e dati mutuati da altre situazioni cliniche (37) applicati in contesti similari (exit site dei cateteri peritoneali e dei CVC) e monitorati negli esiti;

- Valutazione e stimolazione della motivazione professionale degli operatori attraverso la condivisione dei dati con audit periodici (30) e la formazione attivata prima del cambiamento delle pratiche (39). La motivazione a fare bene è stata citata come elemento trainante $(17,19,36)$, come la sua mancanza è stata citata come ragione di alcune previsioni mancate (6);

- Considerazione che l'applicazione della tecnica di puntura a occhiello non è solo "una puntura", ma è una pratica clinica avanzata che necessita di motivazione, abilità e competenze di problem solving nella flessibilità di adattamento al singolo soggetto (12) e concomitante adesione ai criteri di base $(10,31)$.

- Presenza di figure e ruoli professionali di riferimento clinico e gestionale, con competenze avanzate, che hanno attivato, supportato e monitorato il cambiamento nelle pratiche cliniche. Figure specifiche per specifiche popolazioni, come quelle dei pazienti domiciliari, per specifici problemi quali gli accessi vascolari. La presenza di figure professionali specifiche ha reso possibile attivare percorsi di ricerca clinica mirata.

Rispetto alla possibilità di attivare una ricerca nazionale multicentrica, si dovranno accertare le condizioni operative dei centri e, probabilmente, si dovrà richiedere come condizione di partecipazione proprio la definizione di figure di competenza avanzata. Diversamente, il rischio di creare bias, nel caso di studi randomizzati controllati, o di raccogliere dati non confrontabili, nel caso di studi osservazionali, renderebbe inutilizzabili i risultati.

Una sfida che va ben oltre la semplice "puntura a sito costante".

\section{Riassunto}

Negli ultimi 10 anni, vi è stata una notevole produzione di articoli relativi a studi primari e secondari sull'applicazione della tecnica a occhiello, definita, nel gergo degli addetti ai lavori, "buttonholing". Tutti gli studi hanno cercato di verificare, in modo singolo o congiunto, le allettanti promesse del buttonhole: meno dolore, meno aneurismi, minori complicazioni e maggiore facilità di esecuzione, sia per gli infermieri che per i pazienti. Raccogliendo dati su queste aree, con tempistiche, campioni e contesti anche diversi, sono comparse, anche se con bassa o nulla significatività statistica, le complicanze infettive che la letteratura iniziale degli anni ' 80 riportava solo come evenienze possibili ma remote. Il presente elaborato fa riferimento alle ricerche più recenti e significative, alle variabili indagate e agli esiti osservati cercando di evidenziare quali siano le componenti per una pratica clinica in grado di creare esiti positivi per i pazienti e per il personale di assistenza.

\section{Ringraziamenti}

Al Centro di Documentazione Biomedica dell'Ospedale Niguarda, Milano, nelle figure del Dr. De Gregori, della Dr.ssa Claudia Ghezzi e della Dr.ssa Gloria Innocenti, per l'assistenza alla ricerca bibliografica.

Parole chiave: Paziente in emodialisi, Fistola arterovenosa, Puntura a occhiello, Infermiere degli accessi vascolari

Dichiarazione di conflitto di interesse: L'Autore dichiara di non avere conflitto di interessi.

I contenuti del presente articolo sono stati tratti dalla tesi di Laurea Magistrale in Scienze Infermieristiche e Ostetriche, A.A. 2012, di Marisa Pegoraro, relatore Prof. F. Faggiano, Università del Piemonte Orientale, sede di Novara.

Indirizzo degli Autori:

Dr.ssa Marisa Pegoraro

EDTNA/ERCA Filiale Italiana

c/o DIALISI CAL Corsico

Via Travaglia 5

20094 Corsico

marisa_pegoraro@fastwebnet.it 


\section{Bibliografia}

1. Saran R, Pisoni RL, Weitzel WF. Epidemiology of vascular access for hemodialysis and related practice patterns. Contrib Nephrol 2004; 142: 14-28. PMID:14719384.

2. Pisoni RL, Young EW, Dykstra DM, et al. Vascular access use in Europe and the United States: results from the DOPPS. Kidney Int 2002; 61 (1): 305-16. PMID:11786113.

3. Maples D. Nurses' impact on the choiches and longevity of Vascular Access. Nephrol Nurs J 2005; 32 (6): 670-4. PMID:16425812.

4. McCann M, Einarsdottir H, Van Waeleghem JP, et al. Vascular Access Management II: AVF/AVG cannulation techniques and complications. J Ren Care 2009; 35 (2): 90-8. PMID:19432854.

5. Murcutt G. Buttonhole cannulation: should this become the default technique for dialysis patients with native fistulas? Summary of the EDTNA/ERCA Journal Club discussion Autumn 2007. J Ren Care 2008; 34 (2): 101-8. PMID:18498575.

6. Chow J, Rayment G, San Miguel S, et al. A randomised controlled trial of buttonhole cannulation for the prevention of fistula access complications. J Ren Care 2011; 37 (2): 85-93. PMID:21561544.

7. MacRae JM, Ahmed SB, Atkar R, et al. A randomized trial comparing buttonhole with rope ladder needling in conventional hemodialysis patients. Clin J Am Soc Nephrol 2012; 7 (10): 1632-8. PMID:22822010.

8. Struthers J, Allan A, Peel RK, et al. Buttonhole needling of ateriovenous fistulae: a randomized controlled trial. ASAIO J 2010; 56 (4): 319-22. PMID:20418768.

9. Toma S, Shinzato T, Fukui H, et al.A timesaving method to create a fixed puncture route for the buttonhole technique. Nephrol Dial Transplant 2003; 18 (10): 2118-21. PMID:13679489.

10. Marticorena RM, Hunter J, Cook R, et al. A simple method to create buttonhole cannulation tracks in a busy hemodialysis unit. Hemodial Int 2009; 13 (3): 316-21. PMID:19515069.

11. Mott S, Prowant BF. The "touch cannulation" technique for hemodialysis. Nephrol Nurs J 2008; 35 (1): 65-6. PMID:18372765.

12. Figueiredo AE, Viegas A, Monteiro M, et al. Research into pain perception with arteriovenous fistula (avf) cannulation. J Ren Care 2008; 34 (4): 169-72. PMID:19090894.

13. Ball LK, Treat L, Riffle V, et al. A multi-center perspective of the Buttonhole Technique in the Pacific Northwest. Nephrol Nurs J 2007; 34 (2): 234-41. PMID:17486957.

14. Marticorena RM, Hunter J, Macleod S, et al. The salavage of aneurismal fistulae utilizing a modified buttonhole cannulation technique and multiple cannulators. Hemodial Int 2006; 10 (2): 193-200. PMID:16623674.

15. Hashmi A, Cheema MQ, Moss AH. Hemodialysis patients' experience with and attitudes toward the buttonhole technique for arteriovenous fistula cannulation. Clin Nephrol 2010; 74 (5): 346-50. PMID 20979942.

16. Castro MC, Silva Cde F, Souza JM, et al. Arteriovenous fistula cannulation by buttonhole technique using dull needle. J Bras Nefrol 2010; 32 (3): 281-5. PMID:21103692.

17. Silva Gdos S, Silva RA, Nicolino AM, et al. Initial experience with the buttonhole technique in a Brazilian hemodialysis center. J Bras Nefrol 2010; 32 (3):257-62. PMID:21103688.

18. Kim MK, Kim HS. Clinical effects of buttonhole cannulation method on hemodialysis patients. Hemodial Int 2013; 17 (2): 294-9. PMID:22998500.

19. Ward J, Shaw K, Davenport A. Patients' perspectives of constant-site (buttonhole) cannulation for haemodialysis access. Nephron Clin Pract 2010; 116 (2): c123-7. PMID:20502048.

20. Hartig V, Smyth W. "Everyone should buttonhole": a novel technique for a regional Australian renal service. J Ren Care 2009; 35 (3): 114-9. PMID:19689692.

21. Verhallen AM, Kooistra MP, van Jaarsveld BC. Cannulating in haemodialysis: rope-ladder or buttonhole technique? Nephrol Dial Transplant 2007; 22 (9): 2601-4. PMID:17557776.

22. Van Loon MM, Goovaerts T, Kessels AG, et al Buttonhole needling of haemodialysis arteriovenous fistulae results in less complications and interventions compared to the rope-ladder technique. Nephrol Dial Transplant 2010; 25 (1): 225-30. PMID:19717827.

23. Kumbar L. Complications of arteriovenous fistulae: beyond venous stenosis. Adv Chronic Kidney Dis 2012; 19 (3): 195-201. PMID:22578680.

24. Lee T, Barker J, Allon M. Needle infiltration of arteriovenous fistulae in hemodialysis: risk factors and consequences. Am J Kidney Dis 2006; 47 (6): 1020-6. PMID:16731297.

25. Van Loon M, van der Mark W, Beukers N, et al. Implementation of a vascular access quality programme improves vascular access care. Nephrol Dial Transplant 2007; 22 (6): 1628-32. PMID:17400567.

26. Ludlow V. Buttonhole cannulation in hemodialysis: improved outcomes and increased expense--is it worth it? CANNT J 2010; 20 (1): 29-37. PMID:20426358.

27. Hsiao JF, Chou HH, Hsu LA, et al. Vascular changes at the puncture segments of arteriovenous fistula for hemodialysis access. J Vasc Surg 2010; 52 (3): 669-73. PMID:20620004.

28. Ball LK. The buttonhole technique for arteriovenous fistula cannulation. Nephrol Nurs J 2006; 33 (3): 299-304. PMID:16859201.

29. Twardowsky ZJ, Kubara H. Different sites versus constant sites of needle insertion into arteriovenuous fistulas for treatment by repeated dialysis. Dial Transplant 1979; 8: 978-80.

30. Labriola L, Jadoul M. Infectious complications following conversion to buttonhole cannulation. Clin Nephrol 2011; 76 (5): 423. PMID:22000566.

31. Doss S, Schiller B, Moran J, Buttonhole cannulation--an unexpected outcome. Nephrol Nurs J 2008; 35 (4): 417-9. PMID: 18783005.

32. Birchenough E, Moore C, Stevens K, et al. Buttonhole cannulation in adult patients on hemodialysis: an increased risk of infection? Nephrol Nurs J 2010; 37 (5): 491-8. PMID:20973303.

33. Van Eps CL, Jones M, Ng T, et al. The impact of extended-hours home hemodialysis and buttonhole cannulation technique on hospitalization rates for septic events related to dialysis access. Hemodial Int 2010; 14 (4): 451-63. PMID:20955279.

34. Kregness A. Believing in the buttonhole technique. Nephrol News Issues 2008; 22 (12): 36, 40, 42. PMID:19105515.

35. KDOQI; National Kidney Foundation. National Kidney Foundation, NKF 2006. Updates Clinical Practice Guidelines and Recommendations. s.1. : NKF-ISBN 1-931472-22-X.

36. Leitch R, Ouwendyk M, Ferguson E, et al. Nursing issues related to patient selection, vascular access, and education in quoti- 
dian hemodialysis. Am J Kidney Dis 2003; 42 (1 Suppl.): 56-60. PMID:12830445.

37. Nesrallah GE, Cuerden M, Wong JH, Pierratos A. Staphylococcus aureus bacteremia and buttonhole cannulation: long-term safety and efficacy of mupirocin prophylaxis. Clin J Am Soc Nephrol 2010; 5 (6): 1047-53. PMID:20413438.

38. Ball LK, Mott S. How do you prevent indented buttonhole sites? Nephrol Nurs J 2010; 37 (4): 427-8, 431. PMID:20830950.

39. King J. Implementing the buttonhole method using the Biohole peg in a busy dialysis unit: a report of the development of current practice. J Ren Care 2009; 35 (4): 192-200. PMID:19909412.

40. Mott S, Moore H. Kinder, Gentler methods for scab removal in buttonhole access. Nephrol Nurs J 2011; 38 (5): 439-43. PMID:22032006.

41. Dinwiddie LC. Investing in the lifeline: the value of a vascular access coordinator. Nephrol News Issues 2003; 17 (6): 49, 52-3. PMID:12778617.

42. Polkinghorne KR, Seneviratne M, Kerr PG. Effect of a vascular access nurse coordinator to reduce central venous catheter use in incident hemodialysis patients: a quality improvement report. Am J Kidney Dis 2009; 53 (1): 99-106. PMID:18805614.

43. Carlton $D$. The vascular access coordinator role: an interview with Donna Carlton by Betsy King. Nephrol Nurs J 2005; 32 (6): 688-90. PMID:16425819.

44. Waterhouse D. Vascular access: a role for a renal nurse clinician. EDTNA ERCA J 2002; 28 (2): 64-6, 69. PMID:12216845.

45. Guadagno V. L'assistenza infermieristica al paziente in emodialisi: applicazione di modelli organizzativi che esaltano le competenze per migliorare la qualità del servizio. Giornale di Tecniche Nefrologiche e Dialitiche 2012; 24: 1-4. 